\title{
MICHEL COXCIE, PINTOR GRATO A LA CASA DE HABSBURGO*
}

Michel Coxcie (1499-1592), pintor romanista de Brabante, gozó a lo largo de su dilatada carrera del reconocimiento y favor de los Habsburgo; de ello dan testimonio las numerosas obras suyas que hoy ornan los museos y colecciones, muchos españoles, depositarios de los antiguos fondos de la Casa de Austria. Pese al interés relativo suscitado por este artista en la historiografía reciente, los siguientes párrafos esbozan, a la luz de la documentación proporcionada por los archivos, las claves de la fortuna de Coxcie en los círculos cortesanos de la Monarquía Hispánica, con particular atención al entorno de Felipe II, cuya protección se reveló determinante en momentos peliagudos de su vida personal.

Los primeros contactos entre Michel Coxcie y los Habsburgo debieron de producirse gracias a la mediación de Bernard Van Orley, al que se viene considerando su mentor desde los tiempos de Van Mander ${ }^{1}$, y quien fue pintor de corte de las gobernadoras Margarita de Austria y María de Hungría. A la muerte de Van Orley en 1541, Coxcie habría suplido el vacío dejado por el maestro, heredando no sólo sus encargos, sino también el favor de María de Hungría. Hasta tal punto, que pronto empezaría a ser conocido como "schildere der Coninclycke Majesteyt", esto es, "pintor de Su Real Majestad", entendiendo con ello que la gobernadora también era viuda del último rey de Hungría, Luis II Jagellón²; en tales términos se le alude en el pago de unas rentas de 1546 a 1547, las cuales figuran en las cuentas de los pobres de la iglesia de Saint-Géry, hoy desaparecida, y de la colegiata de Santa Gúdula de Bruselas ${ }^{3}$.

En 1550, Coxcie fue nombrado director general de la fábrica de tapices de Bruselas en sustitución de Pieter Coecke van Aelst. En dicho puesto se mantuvo hasta que en 1563 fue reemplazado por Pieter De Kempeneer, pintor que acababa de llegar de Sevilla, en donde había pasado más de quince años y era conocido con el nombre de "Pedro de Campaña"4. Al abandonar la dirección de la fábrica, la asignación anual de 50 florines que el cargo

\section{* ABREVIATURAS:}

A.D.N.: Archives départementales du Nord-Pas de Calais, Lille (Francia).

A.G.S.: Archivo General de Simancas.

A.P.R.M.: Archivo del Palacio Real de Madrid.

${ }^{1}$ Karel Van Mander es autor de un célebre Libro de Vidas de artistas europeos septentrionales, que entronca obviamente con la tradición vasariana: VAN MANDER, K., The lives of the Illustrious Netherlandish and German Painters (edición de Hessel MIEDEMA), vol. I. Doornspijk, Davaco, 1997, p. 292 (f. ${ }^{\circ} 258 v$, 8-13).

2 Van Den Boogert, B. C., "Michiel Coxcie, hofschilder in dienst van het Habsburgse huis", en De Smedt, R. (ed.), Michel Coxcie, pictor regis (1499-1592) [actas del congreso organizado por la Universidad de Malinas el 5 y 6 de junio de 1992], separata de Handelingen van de Koninklijke Kring voor Oudheidkunde, Letteren en Kunst van Mechelen, 2. ${ }^{\circ}$ semestre de 1992. Malinas, 1993, p. 126.

${ }^{3}$ Frankignoulle, E. y Bonenfant, P., "Notes pour servir à l'histoire de l'art en Brabant", Annales de la Société royale d'Archéologie de Bruxelles, n. ${ }^{\circ}$ 39. Bruselas, 1935, pp. 111-119.

${ }^{4}$ Duverger, E., "De Brusselse stadspatroonschilder voor de tapijtkunst Michiel van Cocxyen (ca. 1497-1592)", en DE SMEDT, R. (ed.), op. cit., p. 165. 
comportaba fue traspasada a De Kempeneer, de acuerdo con un documento del 28 de mayo de $1563^{5}$.

Coxcie llegaría a realizar numerosas obras por encargo de María de Hungría. Entre ellas, se incluyen los cartones para las vidrieras de la capilla del Santo Sacramento de la colegiata de Santa Gúdula de Bruselas ${ }^{6}$, así como la copia del Descendimiento de Van der Weyden que fue destinada a sustituir el original en la capilla de la corporación de ballesteros de Nuestra Señora Extramuros de Lovaina. Posteriormente, tanto el original como la copia acabarían por ser enviados a España y en 1566 el cuadro de Coxcie fue emplazado en la capilla del palacio de El Pardo, donde permaneció hasta su traslado a El Escorial en 19397. No obstante, no parece que éste sea el "Otro lienzo en que está pintado el Descendimiento de la cruz, de mano de MASSE MIGUEL, que tiene siete pies y medio de alto y ocho de ancho" que consta como destinado al capítulo del vicario en la primera entrega de El Escorial, de abril de $1574^{8}$, el cual es verosímilmente una segunda copia. Probablemente esta última es la obra por la que Felipe II ordenó pagar al duque de Alba, junto a un cuadro de Santa Cecilia, 220 escudos, según se colige de una carta de 28 de noviembre de 1569 conservada en Simancas: "Miguel de Coixie mi pintor, que esta os dara, me embio los dias passados un quadro del descendimiento de la cruz de Cristo Nuestro Redemptor y otro de Santa Cecilia, tan bien acabados, que me han satisfecho mucho y en consideracion de su trabajo y buena voluntad con que en esto me ha servido tengo por bien que den por ellos dozientos y veinte escudos de a quarenta platas por una vez, (...)"'. Y quizás ésta es la copia del Descendimiento procedente del convento de Los Ángeles de Madrid que se conserva en El Prado con el número 1894 de catálogo y que Elisa Bermejo considera de autor desconocido de comienzos del siglo XVI ${ }^{10}$.

Otro aspecto peor conocido del mecenazgo de María de Hungría son los retratos de miembros de la familia real que Coxcie realizó por encargo suyo. No sólo es seguramente el caso del retrato de Cristina de Dinamarca del Museo de Budapest, realizado en 1545 cuando la joven acababa de enviudar del duque Francisco de Lorena ${ }^{11}$, sino también de cuatro retratos mencionados en un manuscrito proveniente de los archivos de la Cámara de Cuentas de Flandes. En él, se certifica el reembolso que el director de la casa de María de Hungría, Rogier Pathie, obtuvo el 13 de julio de 1554 por la retribución de 280 libras de Flandes pagadas a Michel Coxcie en concepto de un retrato de Carlos V, otro de su mujer Isabel de Portugal, un tercero de Leonor de Austria y otro último de María de Hungría. Cada uno de ellos fue cobrado a 35 escudos: "Au devant dit maistre Rogier Pathie controlleur de l'hostel de la reyne regente en la somme de trois cens quarantehuict livres huict solz dudict pris pour son rembourssement de semblable somme par luy paiee, assavoir 280 semblablez livres, a maistre Michel van Coxien, painctre, pour avoir pourtraict l'empereur, l'imperatrice, la royne tres chretienne

5 Wauters, A., Les tapisseries bruxelloises. Essai historique sur les tapisseries et les tapissiers de haute et de basse-lice de Bruxelles. Bruselas, Culture et Civilisation, 1973, pp. 129-130.

${ }^{6}$ LefÈvre, Pl., "Documents relatifs aux vitraux de Sainte-Gudule, à Bruxelles, du XVI et du XVII ${ }^{\mathrm{e}}$ siècle", Revue belge d'archéologie et d'histoire de l'art, XV, 3/4. Amberes, Académie royale d'archéologie de Belgique, 1945, pp. 117-162; De Crombrugghe, D., Bruxelles. Cathédrale des Saints-Michel-et-Gudule. Vitraux des XVIe et XVII siècles. Bruselas, U.L.B., 2002; VAnden Bemden, Y., "Michel Coxcie, créateur de vitraux", en De SMedt, R. (ed.), op. cit., pp. 141-159.

7 Bermejo Martínez, E., La Pintura de los Primitivos Flamencos en España, t. I. Madrid, CSIC, 1980, p. 106; Ollero Butler, J., "Miguel Coxcie y su obra en España”, AEA, n. ${ }^{\circ}$ 190-191. Madrid, CSIC, 1975, pp. 196-197.

${ }^{8}$ ZARCo CuEvas, J., "Inventario de las alhajas, relicarios, estatuas, pinturas, tapices y otros objetos de valor y curiosidad donados por el rey don Felipe II al Monasterio de El Escorial. Años de 1571 a 1598", Boletín de la Real Academia de la Historia, n. ${ }^{\circ}$ 96. Madrid, 1930, p. 662.

9 A.G.S., Secretaría de Estado, sección: Flandes, Holanda, Bruselas, Roma, negocios extraordinarios de la parte del norte, secretaría provincial de Flandes y Borgoña, contaduría del sueldo, contaduría mayor de cuentas, etc., leg. 544, f. $^{\circ} 202$.

10 Bermejo Martínez, E., op. cit., pp. 106-107.

11 Rose, P., "Christina of Denmark by Michael Coxie", Allen Memorial Art Museum Bulletin, 21, n. ${ }^{0} 1$. Oberlin, otoño de 1963, pp. 29-51. 
et la dicte reine regente, ce a raison de trentecincq escuz chacun tableau"12. Ninguno de estos cuadros se conserva; de haber sido así, hoy conoceríamos mejor esta faceta de retratista real del artista.

La protección de la gobernadora presumiblemente permitió que Michel Coxcie entrara en contacto tanto con Carlos V como con Felipe II. De sus relaciones con el emperador, se tiene un conocimiento más exiguo que el de aquéllas desarrolladas con su hijo. Se sabe que hacia 1550 realizó, de nuevo por mediación de María de Hungría, los cartones para una serie de tapices titulada Victorias de Carlos V sobre los príncipes protestantes y que en ella se incluyó una representación de la batalla de Mühlberg (1547); así lo afirmó el propio pintor en una comparecencia ante la Cámara de Cuentas de Flandes el 21 de noviembre de ese año ${ }^{13}$. Según parece, estos episodios nunca llegaron a ser representados en tapicería y el rastro de los cartones se pierde en el siglo XVI. Por otro lado, en el inventario de los bienes que, procedentes del palacio de Coudenberg de Bruselas, Carlos V llevó consigo a su retiro a Yuste, constan cuatro obras atribuidas a Coxcie: dos Caminos del Calvario, una Crucifixión y una Virgen María ${ }^{14}$.

Es probable que Michel Coxcie conociera a Felipe II durante uno de los dos viajes por los Países Bajos que éste realizó en calidad de príncipe heredero a mediados de siglo; entonces, el futuro monarca debió de contemplar las pinturas de Coxcie expuestas en el castillo de Binche con motivo de las memorables fiestas que allí se celebraron en agosto de 1549, y las vidrieras que, basadas en sus cartones, se acababan de instalar en la capilla del Sacramento de Santa Gúdula. En cualquier caso, con ocasión de su segunda estancia, Felipe II le encargó la copia del políptico del Cordero Místico de San Bavón de Gante, una de sus pinturas más conocidas, que más tarde sería enviada a Madrid ${ }^{15}$. La obra se concluyó en 1559, año en el que está fechada, y para su realización el rey incluso obtuvo de los canónigos de San Bavón que se permitiera a Coxcie trabajar directamente en la capilla Vijd ${ }^{16}$, donde se hallaba el original de los hermanos Van Eyck. Neeffs dice que el pintor cobró por ella 4.000 ducados, si bien no especifica su fuente ${ }^{17}$. El nuevo políptico fue destinado a la capilla del Alcázar de Madrid y en el inventario hecho a la muerte de Felipe II, Pantoja de la Cruz lo tasó en 3.500 ducados $^{18}$. En el inventario del Alcázar del año 1600, figura todavía en la capilla ${ }^{19}$, donde fue restaurado en 1617 y en $1622^{20}$. En 1663, fue sustituido en su emplazamiento por el Pasmo de Sicilia ${ }^{21}$. No se sabe qué fue de la obra durante el siglo XVIII, pero, de hallarse en el Alcázar, sin duda se salvó del gran incendio de 1734. En

12 A.D.N., Recette générale des Finances, registro B 2504, 1554, f. 422 r.

13 STEPPE, J. K., "Vlaams tapijtwerk van de $16^{\mathrm{e}}$ eeuw in spaans koninklijk bezit", en Miscellanea Jozef Duverger. Bij dragen tot de kunstgeschiedenis der Nederlanden, vol. II. Gante, Uitgegeven voor de geschiedenis der textielkunsten, 1968, p. 758, n. 156; PInchart, A., Archives des Arts, Sciences et Lettres [Gante, 1860], vol. III. Bruselas, A.G.R., 1994, p. 199.

${ }_{14}$ Pinchart, A., Tableaux et sculptures de Charles-Quint (1556). Bruselas, Soc. de l'Histoire de Belgique, 1856, p. 4.

15 VAN MANDER, K., op. cit., vol. 1, pp. 60 (f. ${ }^{\circ} 200 \mathrm{v}, 24-37$ ), 67 (f. ${ }^{\circ} 202$ r, 8-15) y 70 (f. ${ }^{\circ} 203$ r, 20-23); VASARI, G., Le Vite de' più eccellenti Pittori, Scultori e Architettori [1550-1568] (edición de Rosanna BetTARINI y Paola BAROCHI), vol. 6. Florencia, Studio per edizioni scelte, 1997, p. 224; Pacheco, F., Arte de la Pintura (edición de Bonaventura Bassegoda i Hugas), Madrid, Cátedra, 1990, pp. 471-472.

${ }^{16}$ Duverger, J., "Kopieën van het "Lam Gods"-retabel van Hubrecht en Jan van Eyck", Bulletin des musées royaux des Beaux-Arts de Belgique. Bruselas, marzo de 1954, pp. 58 y 66: En la documentación, el pintor aparece referido como "meester Michiel Hauschij, scildere van den cueninc van Inghelande" ("maestro Michiel Hauschij, pintor del rey de Inglaterra"), aludiendo así a la condición de rey consorte de Inglaterra de Felipe II tras su matrimonio con María Tudor.

17 Neeffs, E., Histoire de la peinture et de la sculpture à Malines, vol. I, La gilde de Saint-Luc. L'Académie des Beaux-Arts. Les Peintres malinois. Gante, Imprimerie Eugène Vanderhaeghen, 1876, p. 160.

18 Ollero Butler, J., op. cit., p. 196; Duverger, J., op. cit., pp. 66-67.

19 Checa Cremades, F., Felipe II, Mecenas de las Artes. Madrid, Nerea, 1993, p. 154.

20 AA. VV., Felipe II, un monarca y su época. Un príncipe del Renacimiento. Madrid, Soc. Est. para la Conmemoración de los Centenarios de Felipe II y Carlos V, 1998, p. 478.

${ }^{21}$ A.P.R.M., Sección Administrativa, Bellas Artes, pintura, leg. 38, exp. 3. 
el siglo XIX, corrió una suerte desigual: Ceán aún la sitúa en el tránsito del Coro a las Reliquias del Escorial ${ }^{22}$, pero tras la invasión francesa, fue enviada en 1808 a Bruselas, a la sazón también bajo dominación napoleónica, a manos del general Belliard ${ }^{23}$. De allí pasó a dispersarse por las colecciones en las que hoy en día se encuentra dividido: Museos Reales de Bruselas, Alte Pinakothek de Múnich y Gemäldegalerie de Berlín ${ }^{24}$.

De regreso en Castilla, Felipe II comenzó a reclamar cada vez un mayor número de pinturas de Michel Coxcie, a la par que heredaba aquéllas traídas por María de Hungría y Carlos V en su retiro a Yuste tras el fallecimiento de ambos en 1558, muchas de las cuales engrosaron las colecciones de El Escorial desde los primeros momentos en que a él empezaron a afluir obras de arte.

El buen entendimiento entre el monarca y el pintor brabanzón se puede rastrear hasta en un lugar tan remoto como Madeira, en cuya catedral de Funchal se ha detectado, gracias a una restauración reciente, la firma de Coxcie en cuatro cuadros conservados en el altar del Senhor Jesus, con datación de 1581: Santa Ana y San Joaquín, Adoración de los Magos, Presentación de Cristo en el Templo y Huida a Egipto. Se estima que fueron una donación de Felipe II, también Felipe I de Portugal, a la catedral insular ${ }^{25}$.

Coincidiendo con la partida del príncipe de los Países Bajos, en la política de éstos se vio sustancialmente incrementada la ascendencia del obispo de Arras, Antoine Perrenot de Granvelle, conocido como Granvela: en 1559, fue nombrado arzobispo de Malinas y en 1561 recibió la púrpura cardenalicia. Granvela, que destacaba en la Corte por su faceta de mecenas y quien ejercía una labor de bisagra entre los Habsburgo e importantes artistas como Leone Leoni, Antonio Moro o Tiziano, también se interesó por la persona y la obra de Coxcie, al que trataría de favorecer en lo venidero. Él mismo hubo de influir en el propio monarca a la hora de atraer al pintor hacia su órbita, según se desprende de una carta que dirigió a Gonzalo Pérez: "entretanto que aquí estoy me ha mostrado el pintor maestre Miguel, que aquí está, un lienzo (...) para S. M., y á la verdad es muy linda pieza, y tengo por cierto, que puesta en sus bordes bien hechos, sería preciada de cuantos la viesen. El ha deseado que yo avisase de ello, y v. m. lo podrá decir á su magestad, que sé que holgaría de verla; y es este el mesmo pintor que copió la tabla de Gante, y se muestra muy deseoso de servir y de ser empleado y creo que tratándole bien iría a do quiera que su magestad mandase"26.

La estima que Granvela y el rey profesaron por el pintor tuvo que ser, a tenor de los documentos, considerable, puesto que consiguió que ambos intermediaran ante el Papa en persona en un turbio asunto en el que se había visto envuelto uno de sus hijos: Willem Coxcie había emprendido en 1567, quizás por iniciativa de su padre, un viaje de formación a Italia; por el camino debió de entrar en contacto con un grupo de protestantes, tal vez calvinistas de los Países Bajos o luteranos germánicos, y junto a éstos fue hecho prisionero apenas hubo llegado a Roma. Todos ellos fueron procesados por la Inquisición romana y Willem Coxcie fue condenado a pasar diez años en las galeras de Gian Andrea Doria. En ese momento Granvela, que desde 1564 se encontraba en la capital pontificia, intercedió a favor del hijo de Coxcie y consiguió que le fuera reducida la pena a cinco años. Ante la gravedad de la situación, Coxcie decidió escribir al rey a Madrid para implorarle que

22 CeÁn Bermúdez, J. A., Diccionario Histórico de los más ilustres profesores de las Bellas Artes en España [1800] (edición facsímil). Madrid, Istmo, 2001., p. 369.

23 Duverger, J., op. cit., p. 59.

24 AA. VV., Felipe II, un monarca y su época (...) [op. cit.], p. 478.

25 Pereira, A. B., "Descoberta autoria dos panéis do Altar do Senhor Jesus", en Jornal da Madeira. Funchal, 1 de enero de 1997; SERRÃo, Vítor, "La peinture maniériste portugaise, entre la Flandre et Rome, 1550-1620”, en DACOS, N. (ed.), Fiamminghi a Roma 1508-1608. Atti del Convegno Internazionale, Bruxelles 24-25 febbraio 1995. Bollettino d'Arte, suplemento al n. ${ }^{\circ}$ 100. Roma, 1997, p. 272.

${ }^{26}$ Sentenach y Cabañas, N., La pintura en Madrid desde sus origenes hasta el siglo XIX. Madrid, Administración del Boletín de la Sociedad Española de Excursiones, 1907, pp. 23-24. 
interviniera ante Pío V con el fin de obtener la conmutación definitiva de la condena de su hijo; para ello aducía razones como que el joven había dado ostensibles muestras de arrepentimiento o que había sido siempre un católico de recta conducta: "quanto podeys considerar certificandome que antes deste caso fue siem[pre] criado tan catholicamente que se quiso hazer frayle del Carmen, como consta por cierta attestacion que con esta se os mostrara, y despues que fue preso y anda en galeras diz que ha dado notables muestras de arrepentimiento de su error"27. Felipe II decidió responder a las súplicas de su pintor y el 5 de diciembre de 1569 redactó una carta destinada a su embajador en Roma, Juan de Zúñiga ${ }^{28}$, explicando los pormenores del asunto, así como otras dos misivas más dirigidas al cardenal Granvela, en las que daba instrucciones de cómo se había de proceder en el asunto: "Yo os ruego muy affectuosamente que (demas de hazer por vuestra parte con su Sanctidad los buenos officios que convengan) advirtays a Don Juan de Çuñiga mi embaxador de los que os paresciera, que el por la suya y en mi nombre podra hazer para que se consiga esta gracia de su Beatitud (...) y de qualquier buena obra que vos en esto hizieredes al dicho Guillermo Coxie recibire yo mucho contentamiento" 29 . El caso no deja de ser, cuando menos, insólito, a la vez que muy elocuente: que Granvela intercediera en un primer momento para evitar que la pena infligida fuera reducida a la mitad es de por sí un acto de magnanimidad, pero que dos años después el rey se decidiera a influir en la jurisdicción del Sumo Pontífice para obtener el perdón definitivo del hijo de un pintor, es realmente excepcional. Y el hecho es que tales operaciones terminaron por dar sus frutos: Zúñiga y Granvela, asistidos por el cardenal Pacheco, convencieron a Pío V de que absolviera la pena al hijo de Coxcie y el Papa dio instrucciones por carta a Gian Andrea Doria de que así se hiciera. El 14 de junio de 1570, Granvela anunció a Felipe II que Willem Coxcie había sido liberado, narrándole los últimos acontecimientos: "Halo concedido Su Santidad y mandado escrivir cartas a Juan Andrea Doria para que lo ponga en libertad, mas es con condicion que venga a vivir conmigo algun tiempo porque se confirme en la fee y que veamos si realmente queda arrepentido. Si viniere, le recibire de buena gana, pues pienso que en ello servire a Vuestra Majestad viendo la aficion con que manda se solicitte su liberacion"30.

En el panorama de guerra que vivieron los Países Bajos a partir de 1566, Coxcie contó también con el beneplácito del Duque de Alba, gobernador entre 1567 y 1573. Así se desprende de una carta, hoy en Simancas, escrita por Alba a Gabriel de Zayas, secretario del rey, en Bruselas el 13 de abril de $1568^{31}$. De nuevo, se trata de una situación extraordinaria, ya que Coxcie residía entonces en uno de los bastiones de la revuelta: Malinas. Ello no fue óbice para que el pintor obtuviera del Duque la exención de tener que alojar tropas en su casa ${ }^{32}$ o para que consiguiera que le fueran devueltas unas pinturas que le habían sido confiscadas con anterioridad ${ }^{33}$.

En 1589, la buena entente que Coxcie había mantenido durante décadas con el monarca le fue premiada con una renta vitalicia de 2.500 florines anuales, asignables en base a los ingresos comunes de barrios de Amberes y de Lovaina. Así se lo hizo saber Felipe II al entonces gobernador, su sobrino Alejandro Farnesio, mediante carta escrita en El Escorial el 1 de abril de aquel

27 A.G.S., Secretaría de Estado, despachos diversos, libro 78 (1565), 32v.

28 Ibidem.

29 A.G.S. Secretaría de Estado, despachos diversos, libro 78 (1565), 33. La otra carta es prácticamente idéntica: A.G.S. Secretaría de Estado, Negociaciones de Roma, serie 2. ${ }^{a}$, leg. 910, f. ${ }^{\circ}$ 82. De esta última se hizo eco Luis Pérez Bueno, quien confiesa desconocer los detalles del caso: Pérez Bueno, L., "Recomienda Felipe $2 .^{\circ}$ al hijo de su pintor Coxie", A.E.A., t. XX, n. ${ }^{\circ}$ 79. Madrid, CSIC, julio-septiembre de 1947, pp. 254-255.

${ }^{30}$ A.G.S., Secretaría de Estado, negociaciones de Roma, leg. 913, f. ${ }^{\text {os }}$ 68-69.

31 Alba, Duque de [Jacobo Stuart Fitz-James y Falcó] (ed.), Epistolario del III Duque de Alba, Don Fernando Álvarez de Toledo, vol. II, Años 1560-1571. Madrid, 1952, p. 48.

32 NeEfFs, E., op. cit., p. 151: Carta del 27 de diciembre de 1570 del Duque de Alba a la magistratura de Malinas.

33 PInchart, A., op. cit., vol. III. Bruselas, 1994, p. 199. 
año, en la que el rey muestra su preocupación por el estado del pintor, de quien dice que ya había cumplido la edad de 90 años y que "no le es posible ganarse la vida con su arte"34.

La última obra de Coxcie contiene el único retrato de Felipe II conocido pintado por él: se trata del tríptico de la Leyenda de Santa Gúdula, conservado en la antigua colegiata y hoy catedral de Bruselas, que lleva la fecha de 1592. El rey aparece representado en el reverso de uno de los paneles laterales, como un participante más en la procesión del Sacramento que da nombre a la capilla en que se encuentra la obra, en primer término a la izquierda, girando la cabeza y dirigiendo su mirada directamente al espectador. Aparenta una edad muy inferior a los 65 años que entonces contaba: quizás Coxcie guardara el recuerdo de la última vez que lo vio antes de su regreso a Madrid en 1559 o bien se inspiró en otro retrato realizado por aquel entonces. En el aire queda la pregunta de si el propio monarca se encontraba detrás del encargo, del que apenas se tiene información.

Michel Coxcie murió en los primeros meses de 1592; si nos atenemos a lo que dice Van Mander, perdió la vida al caer de un andamio mientras trabajaba en una obra para el ayuntamiento de Amberes $^{35}$. Aun después de su fallecimiento, Felipe II siguió favoreciendo a la familia del pintor: el 26 de agosto de 1596, encargó a su primogénito, Rafael Coxcie, sendos retratos de Isabel Clara Eugenia y de Isabel de Valois, este último póstumo. Ambos fueron pagados dos meses después junto a un tercer retrato hecho por un presunto colaborador llamado Gisbrecht van Veen, al precio de 254 libras y 10 sueldos de Flandes ${ }^{36}$. En agosto de 1595, se le encargarían dos retratos más: uno de la reina de Inglaterra, es de suponer María Tudor, y otro de la reina de Portugal, tal vez Juana de Austria; por ellos recibiría 242 libras y 10 sueldos el 5 de octubre de $1598^{37}$.

VÍCTOR FERNÁNDEZ SORIANO

\footnotetext{
34 Ibidem, vol. II, pp. 320-321.

35 VAN MANDER, K., op. cit., vol. I, p. 295 (f.o 259r, 13-15).

36 A.D.N., Recette générale des Finances, registro B 2758, 1596, f. ${ }^{\text {ss }} 548 \mathrm{v}$ y $549 \mathrm{r}$.

37 A.D.N., Recette générale des Finances, registro B 2770, 1598, f. ${ }^{\circ} 667$.
} 